\title{
ARTICLE OPEN \\ Super square carbon nanotube network: a new promising water desalination membrane
}

\author{
Ligang Sun ${ }^{1}$, Xiaoqiao $\mathrm{He}^{1}$ and Jian $\mathrm{Lu}^{2,3}$
}

Super square (SS) carbon nanotube (CNT) networks, acting as a new kind of nanoporous membrane, manifest excellent water desalination performance. Nanopores in SS CNT network can efficiently filter NaCl from water. The water desalination ability of such nanoporous membranes critically depends on the pore diameter, permitting water molecule permeatration while salt ion obstruction. On the basis of the systematical analysis on the interaction among water permeability, salt concentration limit and pressure on the membranes, an empirical formula is developed to describe the relationship between pressure and concentration limit. In the meantime, the nonlinear relationship between pressure and water permeability is examined. Hence, by controlling pressure, optimal plan can be easily made to efficiently filter the saltwater. Moreover, steered molecular dynamics (MD) method uncovers bending and local buckling of SS CNT network that leads to salt ions passing through membranes. These important mechanical behaviours are neglected in most MD simulations, which may overestimate the filtration ability. Overall, water permeability of such material is several orders of magnitude higher than the conventional reverse osmosis membranes and several times higher than nanoporous graphene membranes. SS CNT networks may act as a new kind of membrane developed for water desalination with excellent filtration ability.

npj Computational Materials (2016) 2, 16004; doi:10.1038/npjcompumats.2016.4; published online 22 April 2016

\section{INTRODUCTION}

Water scarcity remains a highlighted issue worldwide and becomes more serious due to the various factors such as the growth of population, the increasing global warming and consumptions in industry and agriculture. Although $71 \%$ of the earth is covered with water, only $\sim 3 \%$ of the water all over the world is fresh and suitable for human consumption. ${ }^{1}$ In spite of the wide availability of seawater, environmentally friendly and economic purification techniques are needed to produce fresh water from seawater for sustainability. Nowadays, the most widely adopted commercial desalination techniques are electrodialysis, ${ }^{2}$ nanofiltration ${ }^{3}$ and reverse osmosis (RO). ${ }^{4}$ Although ROs, which use high pressure to force water through porous membranes, are believed to have the greatest practical potential, the water permeability is still not satisfactory, meanwhile energy and capital consumption is intensive. In contrast to classical RO membranes, nanoporous membranes can realise fast water permeation. Nanoporous graphene is one kind of nanoporous membranes. Small molecules can pass through them, whereas larger ones cannot. $^{5-7}$ In addition, nanoporous graphene membranes also can be used as selective molecular sieves for gas purification. ${ }^{8,9}$ Therefore, nanoporous graphene is a newly developed membrane for filtration technology. On the other hand, molecular dynamics (MD) studies have predicted ${ }^{10}$ and experiments have demonstrated fast transport of gases ${ }^{11,12}$ and liquids ${ }^{13-16}$ through carbon nanotube (CNT) membranes. The well-aligned CNTs can serve as robust pores in membranes for water desalination and decontamination applications. ${ }^{17-19}$ Nanoporous membranes open a bright future for water desalination technology.
Super CNT networks can be obtained by assembling single-walled CNTs (SWCNTs) into ordered micronetworks, which is first designed for application in composites. ${ }^{20,21}$ Theoretical and experimental studies show that CNTs can be merged covalently using controlled electron irradiation at high temperatures ${ }^{22}$ or atomic welders during heat treatment. ${ }^{23}$ Some kinds of CNT networks have already successfully been made and exhibit special mechanical properties. $^{24-26}$ Along with the production of CNT networks, theoretical models consisting of covalently bonded two- and threedimensional networks have also been developed. ${ }^{27} \mathrm{MD}$ simulations, atomic finite element method, continuum models and molecular structure mechanics method are developed to study super CNTs. ${ }^{28-31}$ Although existing studies have already shown the potential application of super CNT networks in composites, the potential role of such material for water desalination remains unexplored.

In this work, super square (SS) CNT network is selected as a representative for the study on the water desalination ability. To be specific, the SS CNT networks are constructed from SWCNT arms $(6,6)$, denoted as SS@(6,6) in this work. The detailed introduction can be found in section 'Methods'. The computational results indicate that SS@(6,6) can effectively separate salt from water in the desalination system. On the basis of the results obtained from classical MD simulations, optimal plan can be made to filter saltwater with specific salt concentration by controlling the pressure on SS@(6,6). Furthermore, the deformation and failure mode is also discussed under different pressure on SS@(6,6), which cannot be taken into consideration in other MD simulations ${ }^{5-10}$ because the membranes are completely fixed in their systems. Our calculations demonstrate that water molecules

\footnotetext{
${ }^{1}$ Department of Architecture and Civil Engineering, City University of Hong Kong, Kowloon, Hong Kong, China; ${ }^{2}$ Department of Mechanical and Biomedical Engineering, City University of Hong Kong, Kowloon, Hong Kong, China and ${ }^{3}$ Centre for Advanced Structural Materials, City University of Hong Kong, Shenzhen Research Institute, Shenzhen Hi-Tech Industrial Park, Shenzhen, China.

Correspondence: J Lu (jianlu@cityu.edu.hk)

Received 2 November 2015; revised 26 January 2016; accepted 18 February 2016
} 
can flow across SS@(6,6) with very high permeability (from 171 to $421 \mathrm{l} / \mathrm{cm}^{2} /$ day/MPa) while still rejecting salt ions perfectly. This work will be helpful in developing a new kind of excellent nanoporous membrane based on super CNT networks.

\section{RESULTS}

Different filtration stages with the variation of pressure

We first investigate the pressure on SS@(6,6) $\left(P_{s \mathrm{~S}}\right)$ and the distance between the two layers of SS@ $(6,6)\left(d_{\mathrm{ss}}\right)$ during water desalination. The variation of $d_{\mathrm{ss}}$ and $P_{\mathrm{ss}}$ with the increase of time under constant pulling velocity are shown in Figure 1a. There are mainly three stages during the simulation. In stage I, the filtration velocity gradually reduces accompanied by the increase of pressure (green segment). Note that only very small amount of water molecules are filtered across SS@(6,6) in stage I, which is called pre-filtration stage. In stage II, the $P_{\mathrm{ss}}$ keeps in a stable range and the filtration velocity is almost a constant and equal to $v=0.023 \AA / p s$ (orange segment, filtration velocity is equal to $\partial d_{s s} / \partial t$ ), which is called stable pressure filtration (SPF) stage, the corresponding stable pressure is denoted as $P_{c}$. Note that the salt rejection is $100 \%$ in stage II. When the salt concentration reaches a critical value (defined as SPF concentration limit), filtration enters into stage III. The filtration velocity gradually reduces once again, accompanied by the increase of pressure (yellow segment). This stage is named as the ascending pressure filtration (APF) stage. However, the salt ions may pass through SS@ $(6,6)$ with the increase of pressure in stage III. Therefore, some rules can be summarised: (1) filtration velocity can be kept as a constant under $P_{c}$. (2) There is a SPF concentration limit under constant filtration velocity $v$ and corresponding stable pressure $P_{\mathrm{c}}$. (3) High-concentration salt solution beyond the SPF concentration limit can be filtered by gradually increasing pressure provided that no salt ion passes through SS@(6,6). As increasing pressure could induce mechanical problems such as bending, buckling and failure of the SS CNT networks, stage II is an important regime to be paid attention to establish an optimisation criterion. For salt solution with different concentration, we can choose suitable $P_{c}$ to filter it under relative high filtration velocity.

Pressure, concentration limit and water permeability

To investigate the relationship between the stable pressure $P_{\mathrm{c}}$ and the SPF concentration limit, a series of constant pulling velocities

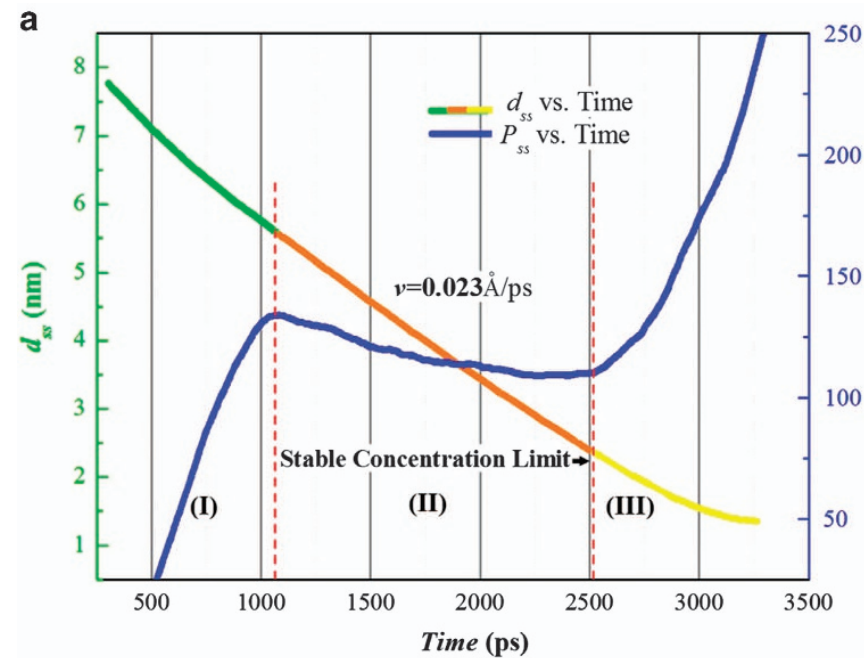

are selected for saltwater desalination. Figure $1 \mathrm{~b}$ shows the relationship between $d_{\mathrm{ss}}$ and $P_{\mathrm{ss}}$ under different constant pulling velocities. All of the cases have a relatively stable pressure $\left(P_{c}\right)$ region corresponding to a series of constant filtration velocities from 0.023 to $0.082 \AA / p s$. Although the velocity is very fast. The filtration velocity can be considered as is equivalent to the water velocity, as the filtration velocity is almost uniform during the stable filtration stage. The water velocity $\sim 2.3$ to $8.2 \mathrm{~m} / \mathrm{s}$ is achievable in real life. In addition, we choose the high filtration velocities to control the pressure on SS@ $(6,6)$ in a desired range $(115-170 \mathrm{MPa})$ for comparison with the applied pressure (100-200 MPa) on nanoporous graphene. ${ }^{6}$ As shown in Figure $1 \mathrm{~b}$, it is obvious that there is a negative correlation between $P_{c}$ and SPF concentration limit. However, there are two kinds of circumstances needed to be distinguished. (1) Low SPF concentration limit induced by low $P_{\mathrm{c}}$ can be further filtered in APF stage. (2) Low SPF concentration limit induced by high $P_{\mathrm{c}}$ cannot be improved in APF stage. Such difference occurs as the salt ions can pass through SS@(6,6) easier under large constant filtration velocities, which can be verified in combination with the data in Table 1 and Figure 1b. For the curve of constant filtration velocity $v=0.032 \AA / p s$, the salt ions pass through $\mathrm{SS} @(6,6)$ when $d_{\mathrm{ss}}=1.6 \mathrm{~nm}$, the corresponding $P_{\mathrm{ss}}$ is $249.2 \mathrm{MPa}$, which is much higher than $P_{\mathrm{c}}$. Furthermore, if the constant filtration velocity is even smaller $(v=0.023 \AA / p s)$, yet no salt ions flow across SS@(6,6) even when $d_{s s}=1.44 \mathrm{~nm}$. However, when the constant filtration velocity is large, salt ions can pass through SS@(6,6) at the very beginning of APF stage. The critical pressure for ion passage is $\sim 175 \mathrm{MPa}$, much smaller than the slow filtration cases that are over $200 \mathrm{MPa}$ (Table 1). So APF is not very suitable for large filtration velocity. Besides, it is discovered that $\mathrm{Na}^{+}$ions always flow across SS@(6,6) earlier than $\mathrm{Cl}^{-}$ions. As the size of $\mathrm{Na}^{+}$ions is smaller than that of $\mathrm{Cl}^{-}$ions, we believe ion size should be an important reason for earlier escape of $\mathrm{Na}^{+}$ions.

Figure $2 a$ further exhibits the relationship between stable pressures $P_{c}$ and SPF concentration limits. Note that the salt rejection is $100 \%$ for all the MD simulation cases under different $P_{\mathrm{c}}$ in Figure 2a. Regions R1 and R2 are consistent to the low SPF concentration limits (as mentioned above) induced by low $P_{c}$ and high $P_{\mathrm{c}}$. If $P_{\mathrm{c}}$ is in region $\mathbf{R} \mathbf{1}$, the filtration limit can be increased by APF, whereas APF hardly works for further filtration if $P_{c}$ is in region $\mathbf{R 2}$. In addition, it can be observed that there exists a critical $P_{\mathrm{c}}^{\mathrm{crt}}$ corresponding to a critical SPF concentration limit.

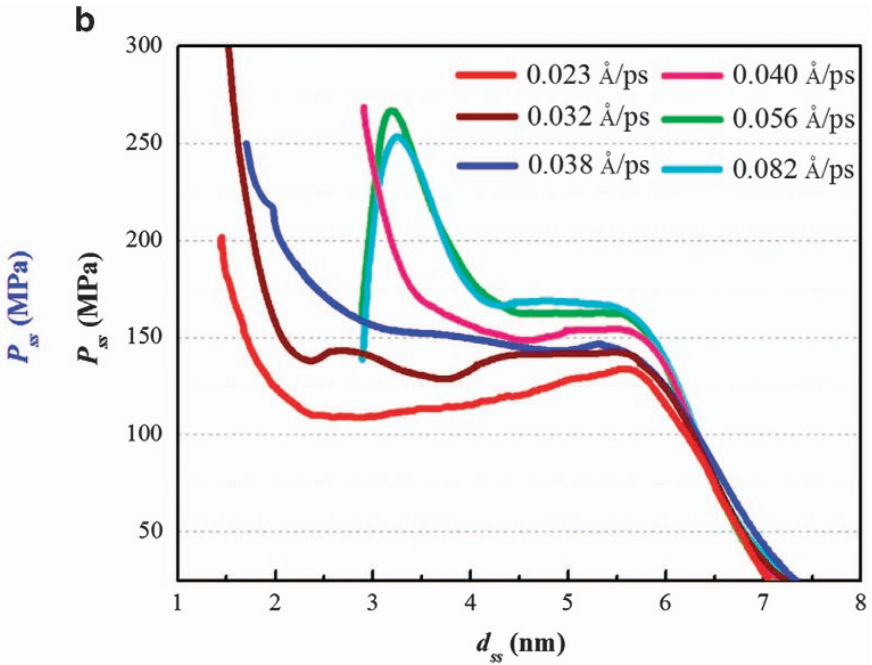

Figure 1. (a) The distance between the two layers of $\mathrm{SS} @(6,6)\left(d_{\mathrm{ss}}\right)$ and the pressure on $\mathrm{SS}(6,6)\left(P_{\mathrm{ss}}\right)$ corresponding to time under constant pulling velocity. (I) The pre-filtration stage. (II) The SPF stage. (III) The APF stage. (b) The relationship between the distance between the two layers of SS@(6,6) $\left(d_{\mathrm{ss}}\right)$ and the pressure on SS $(6,6)\left(P_{\mathrm{ss}}\right)$ under different constant pulling velocities. The velocities from 0.023 to $0.082 \AA / p s$ are the velocities corresponding to different stable pressures. 
Using the curve fitting to the solution of the SPF concentration limit (Figure 2a), an empirical formula is established to describe the relationship between the stable pressure and the SPF concentration limit as follow:

$$
C\left(P_{\mathrm{c}}\right)=\frac{k \mu}{A^{\mathrm{P}} P_{\mathrm{c}}} \cdot \exp \left[h\left(\frac{\ln \left(\frac{P_{\mathrm{c}}}{c_{0}}\right)}{A^{\mathrm{p}}}\right)^{2}\right]
$$

Where $A^{\mathrm{p}}$ is the area of each pore on $\mathrm{SS} @(6,6), \mu$ is the total pore area fraction of one layer of SS@(6,6), and $k=2.69 \times 10^{6}$, $h=-2.78 \times 10^{4}$ and $c_{0}=130.0$ are the fitting parameters.

The optimum pressure for water desalination can be achieved using the formula, depending on two key factors: (1) pore size on the SS CNT network; (2) total pore area fraction on the SS CNT network. It can be seen that: (1) the curve based on equation (1) well coincides with the MD simulation results (Figure 2a). (2) $\mu$ is positive correlated with the SPF concentration limit $(C)$, whereas $A^{\mathrm{p}}$ is negative correlated with the SPF concentration limit (C). It is easy to imagine that if there are more pores on unit area of the SS CNT network, the filtration should be easier with no sacrifice of salt rejection ability, as there is no change of pore size. On the other hand, if the area of each pore $\left(A^{\mathrm{p}}\right)$ is enlarged, the salt rejection ability will reduce and result in the reduction of SPF concentration limit (C). Besides, if the pore size $A_{\mathrm{p}}$ is extremely small, SPF concentration limit $(C)$ approaches infinity. This situation can be imagined as no salt ions can pass through the membrane without pores, nothing to do with the salt concentration. (3) As a new kind of RO membrane, applied pressure is needed to be exerted on the SS CNT networks to overcome osmotic pressure. Thus, if $P_{\mathrm{c}}$ is close to zero, SPF concentration limit $(C)$ converges to zero in equation (1). Furthermore, if $P_{c}$ approaches infinity, the SPF concentration limit $(C)$ also converges to zero in equation (1). Therefore, equation (1) is a reasonable empirical formula to predict the relationship between stable pressures $P_{c}$ and SPF concentration limits. On the basis of the equation (1), the critical stable pressure $P_{c}^{c r t}$ is equal to $124.5 \mathrm{MPa}$ and the critical SPF concentration limit is equal to $99.5 \mathrm{~g} / \mathrm{l}$ for SS@(6,6). Moreover, all the SPF concentration limits in Figure $2 a$ exceed the salinity of seawater ( $35 \mathrm{~g} / \mathrm{l})$. Hence, such kind of the SS CNT networks is with the prospect of seawater purification.

To estimate the water filtration efficiency of SS@(6,6), we calculate the effective water permeability $\left(W_{\mathrm{p}}\right)$ achieved under different $P_{\mathrm{c}}$ (Figure $2 \mathrm{~b}$ ). The water permeability, expressed in liters of output per square centimetre of SS@(6,6) per day and per unit of $P_{\mathrm{c}}$, ranges from 171 to $421 \mathrm{l} / \mathrm{cm}^{2} /$ day/MPa. The filtration efficiency is remarkable. Besides, the permeability scales nonlinearly with $P_{c}$. There exists the relation that the filtration velocity $v$ rises accompanied by the increase of $P_{c}$ (Figure $1 \mathrm{~b}$ ). In addition, we can easily find that the increase of $P_{c}$ results in the improvement of $W_{\mathrm{p}}$ (Figure $2 \mathrm{~b}$ ). Thus, the water permeability should be gradually improved along with the increase of filtration velocity. On the other hand, it is observed that the area of each pore during filtration $\left(A_{1}\right)$ becomes larger than the size after relaxation $\left(A_{0}\right)$. In addition, the variation of pore area $\Delta A$ $\left(\triangle A=A_{1}-A_{0}\right)$ is larger and more intensively under higher $P_{c}$. Therefore, the relationship between $W_{\mathrm{p}}$ and $P_{\mathrm{c}}$ is close related to two key factors: (1) filtration velocity $v$ under different $P_{\text {ci }}$ (2) $\Delta A$ under different $P_{\mathrm{c}}$ during filtration. The combined effect of them results in the nonlinear relationship between $P_{c}$ and $W_{p}$.

Hence, the relatively optimal filtration pressure can be selected with consideration of the relationship among pressure, salt concentration and water permeability discovered in this work as mentioned above.
Table 1. The critical filtration distance $\left(d_{\mathrm{ss}}^{\mathrm{crt}}\right)$ and pressure $\left(P_{\mathrm{ss}}\right)$ for $100 \%$ salt rejection corresponding to different constant filtration velocity

\begin{tabular}{lcccccc}
\hline$v(\AA / \mathrm{ps})$ & 0.023 & 0.032 & 0.038 & 0.040 & 0.056 & 0.082 \\
$d_{\mathrm{ss}}^{\text {crt }}(\mathrm{nm})$ & Null & 1.6 & 2.45 & 3.41 & 3.82 & 4.24 \\
$P_{\mathrm{ss}}(\mathrm{MPa})$ & $>210$ & 249.2 & 173.9 & 174.6 & 184.9 & 167.3 \\
\hline
\end{tabular}

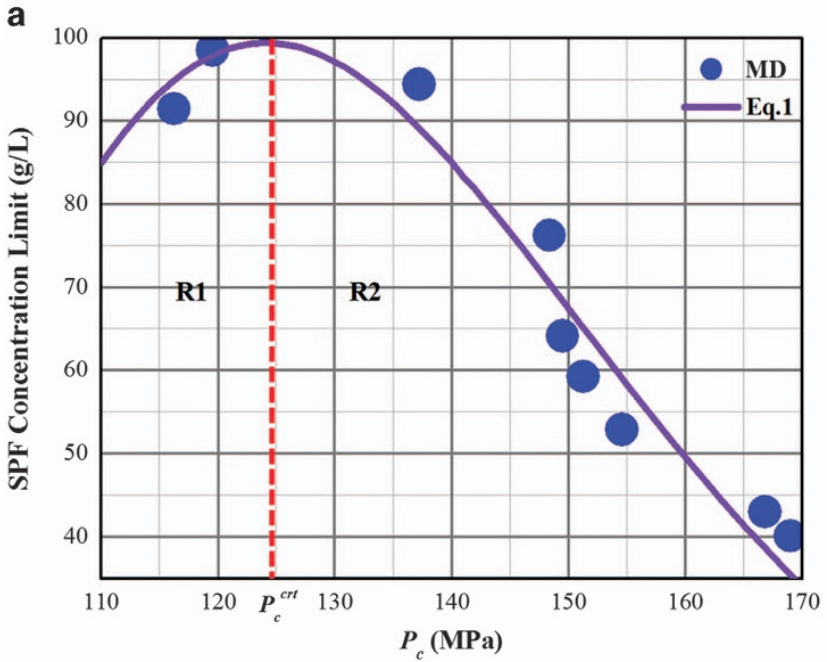

Table 2. Four representative samples with different filtration velocities and the corresponding pressures $\left(P_{c}\right)$

\begin{tabular}{lcccc}
\hline Sample & $I$ & $I I$ & $I I I$ & $I V$ \\
\hline$v(\AA / \mathrm{ps})$ & 0.023 & 0.038 & 0.056 & 0.082 \\
$P_{\mathrm{c}}(\mathrm{MPa})$ & 116.2 & 137.2 & 149.5 & 169 \\
\hline
\end{tabular}

\section{b}

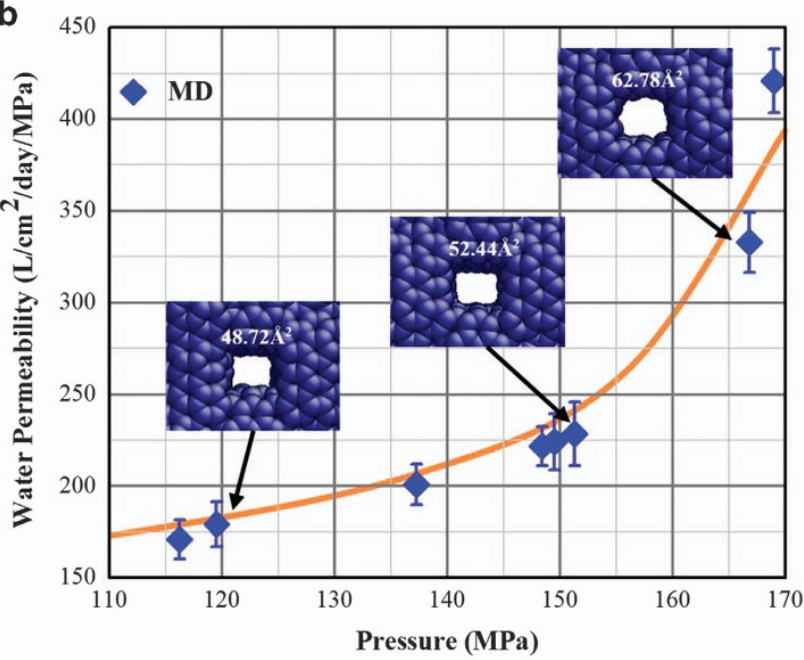

Figure 2. (a) The relationship between stable pressures $\left(P_{c}\right)$ and filtration concentration limits under stable pressure for SS CNT SS@(6,6). Filtration limit can be increased in region R1, whereas cannot be increased in region R2. There exists a critical stable pressure $P_{c}^{\text {crt }}$ corresponding to a critical stable pressure filtration concentration limit. $(\mathbf{b})$ The nonlinear relationship between stable pressures $\left(P_{c}\right)$ and water permeability for SS CNT SS@(6,6). Error bars represent the s.d. of pressures in the stable pressure filtration stage. The illustrations for nanopore size in CNT SS@(6,6) under different pressure. The pore sizes vary more intensively under higher pressure. 

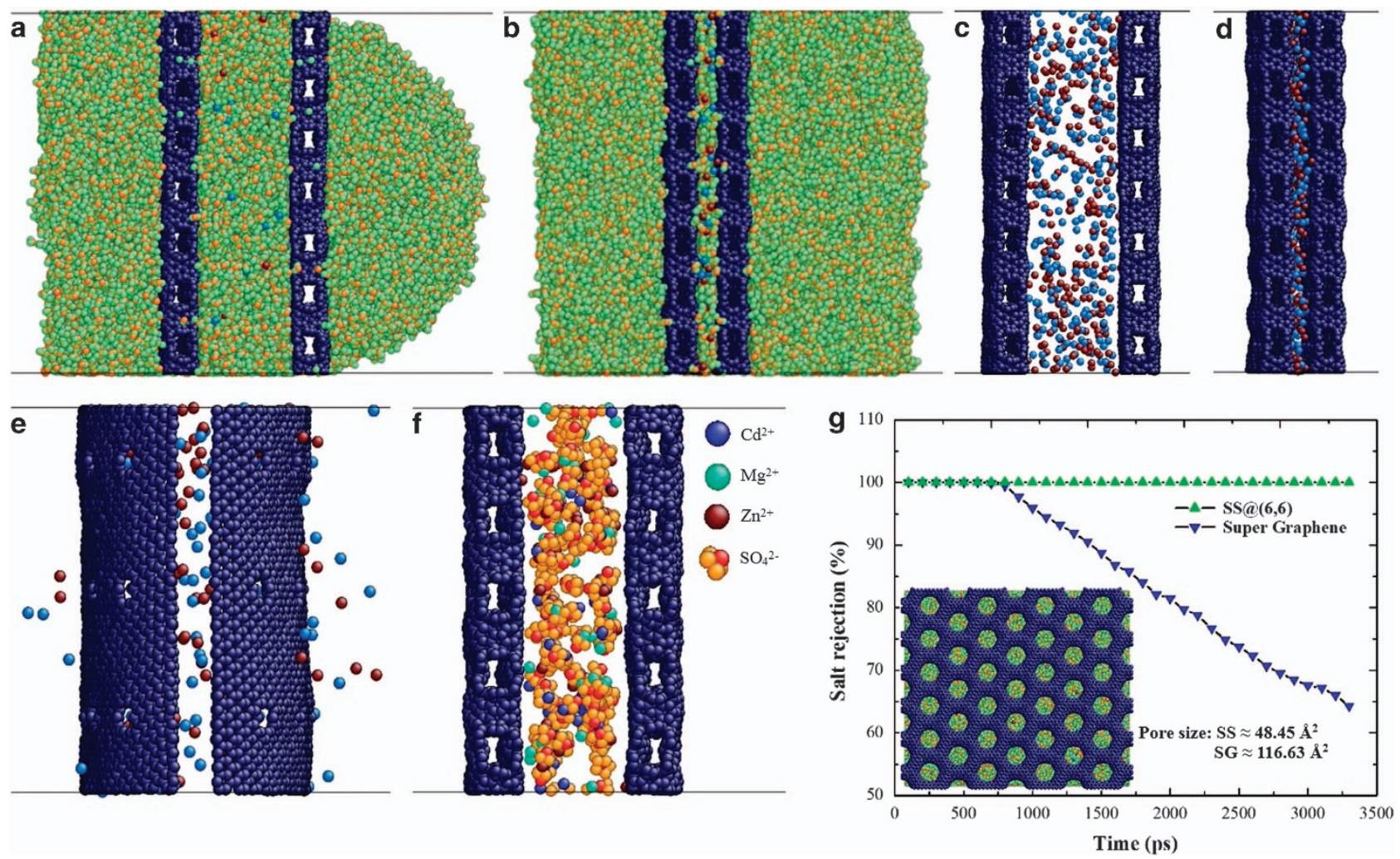

Figure 3. (a, b) The snapshots of SS@(6,6) during water desalination at stable and ascending pressure filtration stage, respectively, under filtration velocity $v=0.023 \AA / p s$. (c, d) The corresponding configuration excluding water molecules in $\mathbf{a}$, $\mathbf{b}$. (e) Salt ions pass through deformed nanoporous graphene during water desalination under filtration velocity $v=0.023 \AA / p s$. (f) Heavy ions filtration by SS@(6,6). (g) The comparison of salt rejection ability between SS@ $(6,6)$ and super graphene.

Structure evolution of SS@(6,6) during water desalination

Four representative samples with different filtration velocities and corresponding pressures $\left(P_{\mathrm{c}}\right)$ are selected to analyse the nanostructure evolution during water desalination (Table 2). Figure 3 shows the nanostructure evolution of sample I during water desalination compared with nanoporous graphene. Figure $3 a$ is one of the pictures obtained during water desalination at SPF stage and Figure $3 \mathrm{c}$ is the corresponding figure in which water molecules are invisible. Figure $3 \mathrm{~b}$ is one of the pictures obtained during water desalination at APF stage and Figure $3 \mathrm{~d}$ is the corresponding figure only showing salt ions. It can be easily observed that the salt ions can be strictly filtered by SS@(6,6). Although the nanoporous graphene has the similar pore size and the same loading procedure as SS@(6,6), the salt ions cannot be impeded by it due to easier mechanical deformation of graphene under compression (Figure 3e). Moreover, the perfect heavy ions rejection ability $\left(\mathrm{Cd}^{2+}, \mathrm{Mg}^{2+}\right.$ and $\left.\mathrm{Zn}^{2+}\right)$ of $\mathrm{SS} @(6,6)$ is also verified by similar filtration process (Figure $3 f$ ). Thus, SS CNT network has more excellent mechanical properties acting as a kind of nanoporous membrane compared with nanoporous graphene. On the other hand, the low SPF concentration limit can be improved in APF stage that is consistent with the $\mathrm{R} 1$ region in Figure $2 a$, as the filtration velocity in sample $I$ is relatively slow. To improve the permeability of SS@(6,6), the filtration velocity is increased by raising $P_{c}$. However, increasing pressure could induce mechanical problems such as bending, local buckling and failure of the SS CNT networks (Figure 4). All these deformations occur in the APF stage through observation of nanostructure evolution during filtration. In addition, the faster the filtration velocity, the sooner the deformations occur in APF stage. The pore size is enlarged after bending or local buckling. Thus, the salt ions can flow across SS@(6,6) in the APF stage and SS@(6,6) lose the filtration effectiveness. Note that the salt ions can pass through SS@(6,6) only when it deforms. Hence, even better filtration capability can be achieved by improving the strength of SS CNT networks through structure design. As the deformation and failure mode of the SS CNT networks is affected by the chiralities and sizes of the SWCNTs, ${ }^{32}$ they can be taken into consideration to design the SS CNT networks with higher strength in the future study.

Outstanding water permeability and salt rejection ability

Overall, our results indicate that the SS CNT networks could act as a high-permeability desalination membrane with guarantee of $100 \%$ salt rejection and water passage. High-concentration salt solution can be purified efficiently by the SS CNT networks. Moreover, to illustrate the water permeability and salt rejection ability of the SS CNT networks, the water desalination performance of the SS@(6,6) from $P_{\mathrm{c}}=116.2$ to $169 \mathrm{MPa}$ is plotted along with the performance of RO membranes ${ }^{33}$ and nanoporous graphenes $^{6}$ in Figure 5. Note that although the MD simulation method adopted in our study is different from most of other studies, $^{5-10}$ we only compare the data in SPF stage (Figure 2b) with other membranes. As the filtration velocity in SPF stage is almost uniform under constant stable pressure, we think the pressure on membranes can approximately be regarded as the applied hydrostatic pressure. Exactly as the performance of nanoporous graphenes, the salt rejection of SS@ $(6,6)$ maintains at $100 \%$, both are superior than conventional RO membranes. On the other hand, the water permeability of SS@ $(6,6)$ is several orders of magnitude higher than RO membranes and several times higher than nanoporous graphene. It should be emphasised that the water permeability of SS@(6,6) can be improved by increasing pressure with no sacrifice of salt rejection (Figure $2 b$ ). 
a

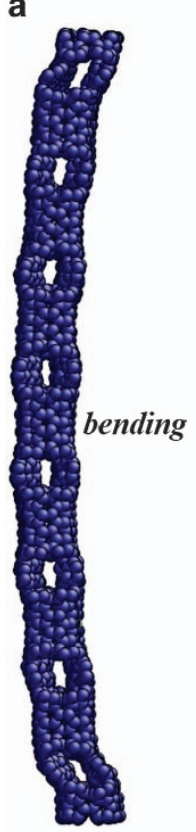

b

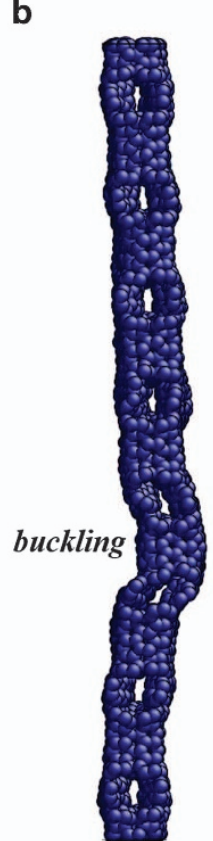

C

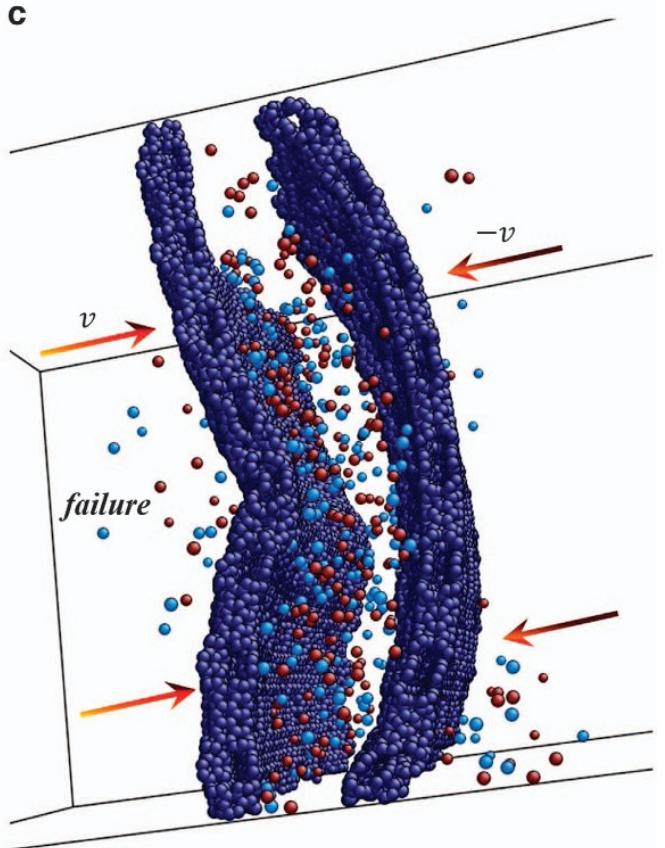

Figure 4. (a) Bending of SS@(6,6) in sample II with filtration velocity $v=0.038 \AA / \mathrm{ps}$ in ascending pressure filtration (APF) stage. (b) Local buckling of SS@(6,6) in sample III with $v=0.056 \AA$ /ps in APF stage. (c) Bending and buckling combined failure of SS@(6,6) in sample IV with $v=0.082 \AA / p$ s in APF stage. A lot of salt ions pass through deformed SS@(6,6).
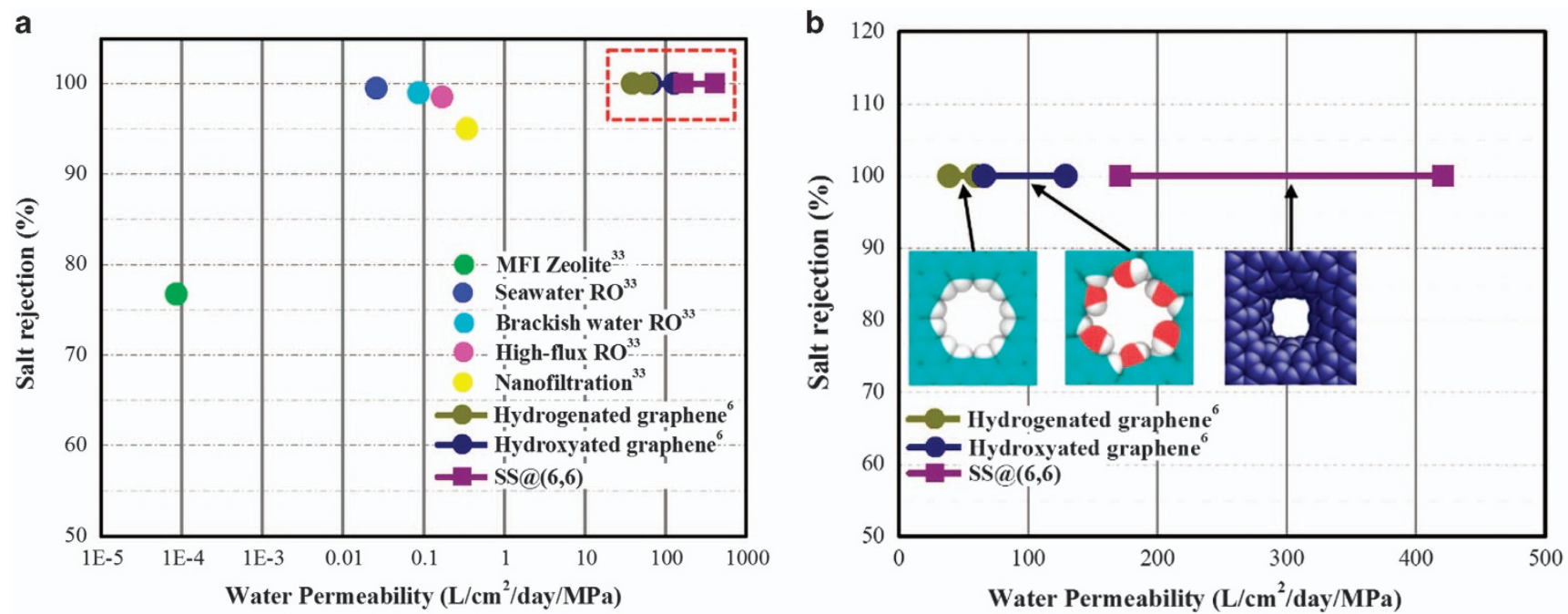

Figure 5. Performance illustration for SS@(6,6) versus existing technologies. The data is adapted from Pendergast et al. ${ }^{33}$ and Cohen-Tanugi et al. $^{6}$ (a) The water permeability of passivated nanoporous graphene ${ }^{6}$ and SS@ $(6,6)$ is expressed by the interval based on the simulation results. The SS@ $(6,6)$ examined in this work could fully reject salt ions with a water permeability several orders of magnitude higher than commercial RO and also have better performance than functionalised nanoporous graphene membranes. (b) The amplified red region in a clearly exhibits the outstanding water permeability of SS@(6,6) compared with nanoporous graphene membranes.

Moreover, the functionalisation by hydrophilic chemical group (hydroxyl, $\mathrm{OH}-$ ) instead of hydrophobic ones (hydrogen atoms, $\mathrm{H}-$ ) on nanoporous graphene can roughly double the water flux. ${ }^{6}$ We think sidewall functionalisation of SS CNT networks to improve hydrophilicity could be an alternative to further improvement the performance of water desalination since both CNTs and CNT networks are chemically hydrophobic. ${ }^{34}$ Therefore, the SS CNT networks could be a new kind of nanoporous membrane possessing outstanding water permeability.

Regarding the question of whether the SS CNT networks could be practical for commercial-scale desalination, we think that the fabrication of well-aligned SS CNT networks with the extremely narrow pore size is a main challenge. The well-aligned SS CNT networks with the strict controlled pore size and shape are not achievable due to the limitation of the current fabrication technology. Nowadays, the experimentally available CNT networks ${ }^{22-26}$ are composed of a random distribution of pores, in both size and geometric shape. The randomness of pore size will inevitably result in the existence of unfavourable large pores. As shown in Figure 3g, the super graphene CNT network is developed and tested by MD simulation. The worse salt rejection is observed due to the enlargement of the pore size. Thus, it is essential to fabricate well-aligned networks with the uniform pore size for practical application in the future. Although challenging, 

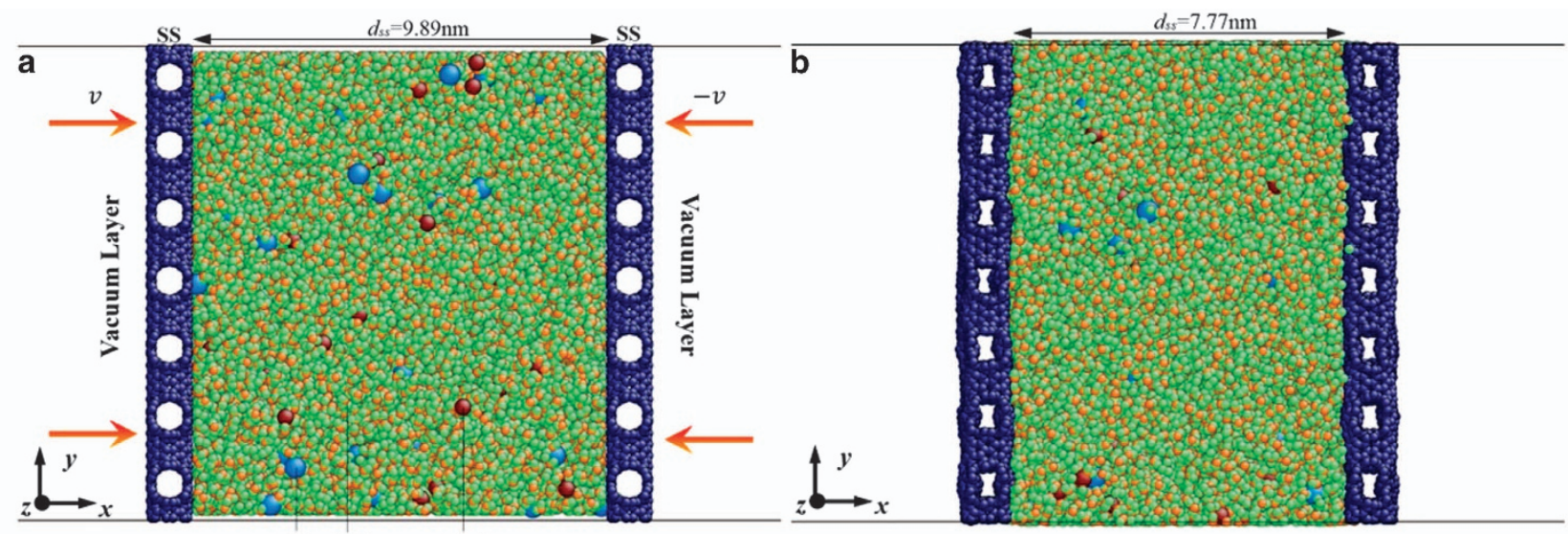

$\mathrm{Cl}^{-} \mathrm{H}_{2} \mathrm{O}$
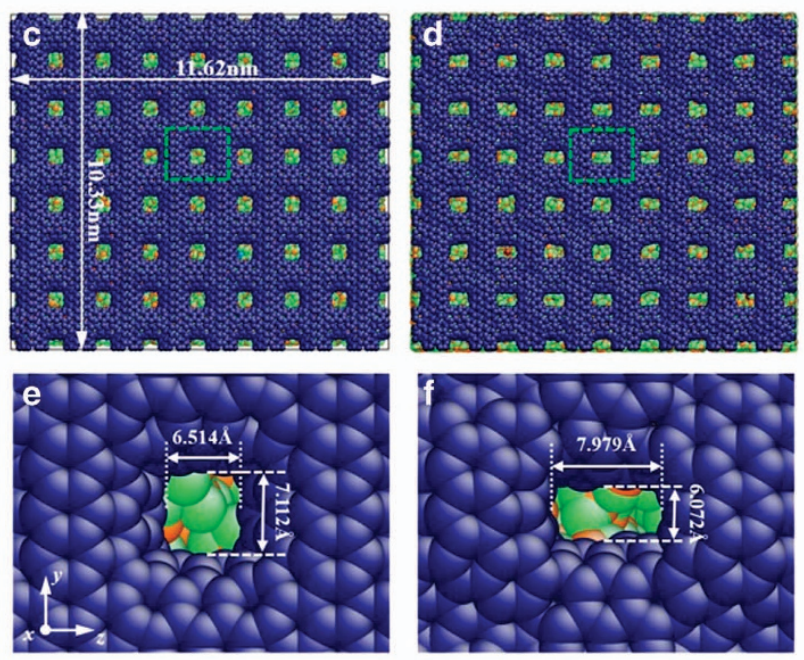

g
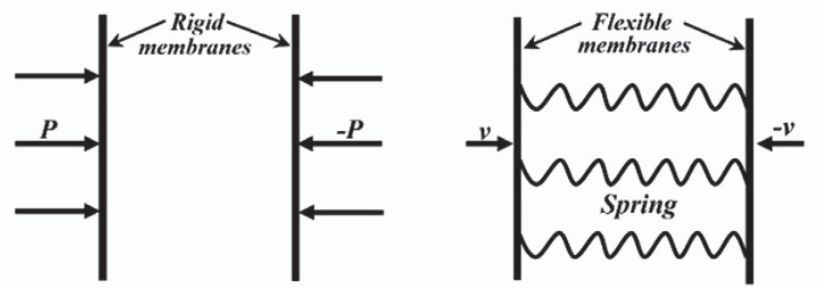

Figure 6. (a) The initial configuration of SS@(6,6) and saltwater. (b) The energy minimised and relaxed configuration of a. (c, d) Side views of a, b. (e, f) Pore sizes of $\mathbf{c}$, d on SS@(6,6). (g) Schematic diagrams of two different simulation methods: the rigid membranes with pressure exerted on them (left) and the flexible membranes under traction by springs (right).

we believe it can be overcome and the CNT networks could offer important advantages over existing RO technology.

\section{DISCUSSION}

Acting as a new kind of nanoporous membrane, the SS CNT networks are demonstrated possessing excellent water permeability and salt rejection ability in our simulations. The water permeability is several orders of magnitude higher than the conventional RO membranes and also several times higher than nanoporous graphene membranes. We find that the water permeability with guarantee of $100 \%$ salt rejection is both affected by the salt concentration of water and the pressure on the SS@(6,6). To systematically describe the interaction among pressure, salt concentration and water permeability, the following two efforts are made: (1) an empirical formula is developed to describe the relationship between pressure and concentration limit; (2) the nonlinear relationship between pressure and water permeability is uncovered. Thus, optimal plan can easily be made to efficiently filter saltwater with specific salt concentration by controlling the pressure on the SS@ $(6,6)$. On the other hand, steered MD simulation method is first adopted to analyse the mechanical behaviour of membranes during water desalination, which cannot be realised due to the limitation of methods adopted by other researchers..$^{5-10}$ On the basis of this method, we discover that the bending and buckling of SS@(6,6) under high pressure, which results in enlargement of the pore size, is the main reason for salt ions passing through SS@(6,6). Thus, better filtration capability can be achieved by improving the strength of SS CNT networks through structure design and optimising the pore size in the SS CNT networks. Therefore, a new kind of nanoporous membrane is studied in this work for water desalination that yields significant improvement over existing technological methods. We expect that our study will promote the understanding of the excellent performance of well-designed SS CNT networks for water desalination technology.

\section{MATERIALS AND METHODS}

All the MD simulations are performed using the LAMMPS package. ${ }^{35}$ Two layers of the SS CNT network are constructed from SWCNT arms $(6,6)$, denoted as SS@(6,6) (Figure 6). The X junction is built by joining four SWCNTs at an angle of $90^{\circ}$ using pentagons and heptagons. Thus, uniformly distributed nanopores are fabricated in SS@(6,6) (Figure 6c) with 25,000 water molecules, $250 \mathrm{Na}^{+}$ions and $250 \mathrm{Cl}^{-}$ions between the two SS@ $(6,6)$ layers, corresponding to the salt concentration of $37.125 \mathrm{~g} / \mathrm{l}$ (similar to the salinity of seawater $35 \mathrm{~g} / \mathrm{l})$. The SS@ $(6,6)$ has dimensions of $11.62 \times 10 \mathrm{~nm}$ (Figure 6c). The periodic boundary condition is imposed along $X, Y$ and $Z$ directions with the two vacuum layers along $X$ direction. CHARMM force field ${ }^{36}$ is adopted to model the interactions between the atoms in the system. Water is modelled by TIP3P potential ${ }^{37}$ and interactions between the atoms are modelled using Lennard-Jones and Coulombic terms. 
Steered MD method ${ }^{38}$ is adopted to simulate desalination process under constant pulling velocity. Note that the constant pulling velocity represents the moving speed of equilibrium position of the spring attached on the SS@(6,6), thus the SS@ $(6,6)$ are pulled under a traction force $F=k \Delta x$, where $k$ is the spring constant and is set to $50 \mathrm{kcal} / \mathrm{mol} / \AA, \Delta x$ is equal to $L-L_{0}, L$ and $L_{0}$ are the actual length and equilibrium length of the spring respectively. As the SS@ $(6,6)$ endure resistance from salt solution, the filtration velocity should be slower than the constant pulling velocity. Moreover, unlike other methods in which the rigid condition is applied on the membranes,,$^{5-10}$ the SS@ $(6,6)$ atoms are not held fixed during the steered MD simulation, so the nanostructure evolution of SS@(6,6) under compression can be detected (Figure $6 \mathrm{~g}$ ). Besides, the salt concentration between SS@ $(6,6)$ changes continuously during the desalination process. We can easily know the performance of SS@(6,6) for water desalination with specific salt concentration under different pressure according to Figure 1. Thus, the desalination process affected by salt concentration can also be analysed.

Initially, the created system is subjected to energy minimisation using the conjugate gradient method, and then relaxed in the Nose/Hoover isobaric-isothermal ensemble (NPT) under both the pressure 0 bar and the desired temperature $(300 \mathrm{~K})$ with an initial Gaussian velocity distribution consistent with the temperature for $300 \mathrm{ps}$. As shown in Figure 6 , the distance between the two layers of SS@(6,6) changes from $d_{\mathrm{ss}}=9.89$ to $7.77 \mathrm{~nm}$ after relaxation. The pore size changes from 46.33 to $48.45 \AA^{2}$. Note that there exists trivial size difference among the pores on the relaxed SS@(6,6). Hereafter, desalination simulation is executed along $X$ direction under a canonical ensemble (NVT). The total simulation time is $1,500-3,500 \mathrm{ps}$ with a time step of $1 \mathrm{fs}$. A series of constant pulling velocity $v$ are selected to simulate the desalination process. The average stress of all the carbon atoms is calculated during the simulation to detect the pressure on SS@ $(6,6)\left(P_{\mathrm{ss}}\right)$. The distance $d_{\mathrm{ss}}$ is also recorded to calculate the filtration velocity and salt concentration variation.

\section{ACKNOWLEDGEMENTS}

The financial support from the Research Grants Council of the Hong Kong Special Administrative Region of China under grants (CityU-114111) is gratefully acknowledged. J.L. acknowledges the financial supports provided by the Grant 2012CB932203 of the National Key Basic Research Program of the Chinese Ministry of Science and Technology and from the Croucher Foundation CityU9500006.

\section{CONTRIBUTIONS}

J.L. and X.H. conceived the project and provided guidance. L.S. performed the theoretical computations, analysis and wrote the manuscript. J.L. and X.H. also reviewed the manuscript and offered helpful suggestions during the work.

\section{COMPETING INTERESTS}

The authors declare no conflict of interest.

\section{REFERENCES}

1. Gleick P. H. (ed.). Water in Crisis: A Guide to the World's Fresh Water Resource (Oxford Univ. Press, 1993).

2. Davis T. A. \& Porter M. C. (eds). Electrodialysis (Noyes Publications, 1990).

3. Pontie, M., Derauw, J. S., Plantier, S., Edouard, L. \& Bailly, L. Seawater desalination: nanofiltration-a substitute for reverse osmosis? Desalin. Water Treat. 51, 485-494 (2013)

4. Fritzmann, C., Löwenberg, J., Wintgens, T. \& Melin, T. State-of-the-art of reverse osmosis desalination. Desalination 216, 1-76 (2007).

5. Wang, E. N. \& Karnik, R. Water desalination: Graphene cleans up water. Nat. Nanotechnol. 7, 552-554 (2012).

6. Cohen-Tanugi, D. \& Grossman, J. C. Water desalination across nanoporous graphene. Nano Lett. 12, 3602-3608 (2012).

7. Konatham, D., Yu, J., Ho, T. A. \& Striolo, A. Simulation insights for graphene-based water desalination membranes. Langmuir 29, 11884-11897 (2013).

8. Hauser, A. W. \& Schwerdtfeger, P. Methane-selective nanoporous graphene membranes for gas purification. Phys. Chem. Chem. Phys. 14, 13292-13298 (2012).

9. Koenig, S. P., Wang, L. D., Pellegrino, J. \& Bunch, J. S. Selective molecular sieving through porous graphene. Nat. Nanotechnol. 7, 728-732 (2012).

10. Hummer, G., Rasaiah, J. C. \& Noworyta, J. P. Water conduction through the hydrophobic channel of a carbon nanotube. Nature 414, 188-190 (2001).
11. Skoulidas, A. I., Ackerman, D. M., Johnson, J. K. \& Sholl, D. S. Rapid transport of gases in carbon nanotubes. Phys. Rev. Lett. 89, 185901 (2002).

12. Holt, J. K. et al. Fast mass transport through sub-2-nanometer carbon nanotubes. Science 312, 1034-1037 (2006).

13. Majumder, M., Chopra, N., Andrews, R. \& Hinds, B. J. Nanoscale hydrodynamics: enhanced flow in carbon nanotubes. Nature 438, 44-44 (2005).

14. Wang, Z. et al. Polarity-dependent electrochemically controlled transport of water through carbon nanotube membranes. Nano Lett. 7, 697-702 (2007).

15. Zhou, J. J., Noca, F. \& Gharib, M. Flow conveying and diagnosis with carbon nanotube arrays. Nanotechnology 17, 4845-4853 (2006).

16. Whitby, M. \& Quirke, N. Fluid flow in carbon nanotubes and nanopipes. Nat. Nanotechol. 2, 87-94 (2007).

17. Elimelech, M. \& Phillip, W. A. The future of seawater desalination: energy, technology, and the environment. Science 333, 712-717 (2011).

18. Hughes, Z. E., Shearer, C. J., Shapter, J. \& Gale, J. D. Simulation of water transport through functionalized single-walled carbon nanotubes (SWCNTs). J. Phys. Chem. C 116, 24943-24953 (2012).

19. Das, R., Ali, M. E., Abd Hamid, S. B., Ramakrishna, S. \& Chowdhury, Z. Z. Carbon nanotube membranes for water purification: A bright future in water desalination. Desalination 336, 97-109 (2014).

20. Song, Y. S. \& Youn, J. R. Influence of dispersion states of carbon nanotubes on physical properties of epoxy nanocomposites. Carbon 43, 1378-1385 (2005).

21. Coleman, J. N., Khan, U., Blau, W. J. \& Gun'ko, Y. K. Small but strong: a review of the mechanical properties of carbon nanotube-polymer composites. Carbon 44 1624-1652 (2006).

22. Terrones, M. et al. Molecular junctions by joining single-walled carbon nanotubes. Phys. Rev. Lett. 89, 075505 (2002).

23. Endo, M. et al. Atomic nanotube welders: boron interstitials triggering connections in double-walled carbon nanotubes. Nano Lett. 5, 1099-1105 (2005).

24. Snow, E. S., Novak, J. P., Campbell, P. M. \& Park, D. Random networks of carbon nanotubes as an electronic material. Appl. Phys. Lett. 82, 2145-2147 (2003).

25. Hall, L. J. et al. Sign change of Poisson's ratio for carbon nanotube sheets. Science 320, 504-507 (2008).

26. Ma, W. J. et al. Directly synthesized strong, highly conducting, transparent single-walled carbon nanotube films. Nano Lett. 7, 2307-2311 (2007).

27. Romo-Herrera, J. M., Terrones, M., Terrones, H., Dag, S. \& Meunier, V. Covalent 2D and 3D networks from 1D nanostructures: designing new materials. Nano Lett. 7, 570-576 (2007).

28. Qin, Z., Feng, X. Q., Zou, J., Yin, Y. J. \& Yu, S. W. Superior flexibility of super carbon nanotubes: molecular dynamics simulations. Appl. Phys. Lett. 91 043108 (2007).

29. Liu, B., Huang, Y., Jiang, H., Qu, S. \& Hwang, K. C. The atomic-scale finite element method. Comput. Methods Appl. Mech. Eng. 193, 1849-1864 (2004)

30. Wang, M., Qiu, X. M., Zhang, X. \& Yin, Y. J. Equivalent parameter study of the mechanical properties of super carbon nanotubes. Nanotechnology 18, 295708 (2007).

31. Li, Y., Qiu, X. M., Yin, Y. J., Yang, F. \& Fan, Q. S. The elastic buckling of super-graphene and super-square carbon nanotube networks. Phys. Lett. A 374, 1773-1778 (2010).

32. Liu, X., Yang, Q. S., He, X. Q. \& Mai, Y. W. Molecular mechanics modeling of deformation and failure of super carbon nanotube networks. Nanotechnology 22, 475701 (2012).

33. Pendergast, M. M. \& Hoek, E. M. V. A review of water treatment membrane nanotechnologies. Energy Environ. Sci. 4, 1946-1971 (2011).

34. Scarselli, M. et al. Applications of three-dimensional carbon nanotube networks. Beilstein J. Nanotechnol. 6, 792-798 (2015).

35. Plimpton, S. Fast parallel algorithms for short-range molecular dynamics. J. Comput. Phys. 117, 1-19 (1995).

36. MacKerell, A. D. et al. All-atom empirical potential for molecular modeling and dynamics studies of proteins. J. Phys. Chem. B 102, 3586-3616 (1998).

37. Jorgensen, W. L., Chandrasekhar, J., Madura, J. D., Impey, R. W. \& Klein, M. L. Comparison of simple potential functions for simulating liquid water. J. Chem. Phys. 79, 926-935 (1983).

38. Izrailev S. et al. (eds). Computational Molecular Dynamics: Challenges, Methods, Ideas, 39-65 (Springer-Verlag, 1998)

This work is licensed under a Creative Commons Attribution 4.0 International License. The images or other third party material in this article are included in the article's Creative Commons license, unless indicated otherwise in the credit line; if the material is not included under the Creative Commons license, users will need to obtain permission from the license holder to reproduce the material. To view a copy of this license, visit http://creativecommons.org/licenses/ by/4.0/ 